\title{
Composition lipidique des spermatozoides humains et susceptibilité au stress oxydant avant et après migration dans le mucus cervical
}

\author{
Nozha CHAKROUN FEKI1, Patrice THEROND2, Pierre JOUANNET1, Jacques AUGER1 \\ 1 Service de Biologie de la Reproduction, Hôpital Cochin, Paris \\ 2 INSERM unité 347, Hôpital de Bicêtre, Laboratoire de Biochimie, Hôpital de Bicêtre, Le Kremlin-Bicêtre
}

\begin{abstract}
RESUME
L'intérêt porté aux effets physiopathologiques des dérivés actifs de l'oxygène (DAO) dans la fertilité masculine est de plus en plus important. Le spermatozoïde humain est une cellule très vulnérable au stress oxydant compte tenu de sa richesse en acides gras polyinsaturés. L'importance des dommages oxydatifs dans les spermatozoïdes dépend surtout de la capacité des fluides environnants à arrêter la chaîne de peroxydation lipidique membranaire. Les secrétions des tractus génitaux masculins et féminins constituent des microenvironnements qui jouent un rôle déterminant dans l'expression du pouvoir fécondant des spermatozoïdes via des échanges avec la membrane plasmique, et assurent une protection vis-à-vis d'un stress $0 x y$ dant en maintenant l'équilibre de la balance prooxydants/antioxydants. Le déséquilibre de cette balance dans l'épididyme ou dans le sperme éjaculé, induit plusieurs dysfonctions spermatiques. En revanche, il existe de très rares données concernant les conséquences d'un stress oxydant au niveau du tractus génital féminin sur les caractéristiques spermatiques.
\end{abstract}

Après avoir abordé, sous forme d'une revue bibliographique, les modifications de la composition lipidique des spermatozoïdes au cours de leur maturation et les effets physiopathologiques des DAO sur les fonctions des spermatozoïdes humains, nous rapportons nos résultats concernant les modifications de la composition lipidique des spermatozoïdes humains après migration dans le mucus cervical et l'influence de la qualité de ce dernier sur les caractéristiques des spermatozoïdes sélectionnés après migration.
Nous avons démontré une baisse des lipides dans les spermatozoïdes humains après migration dans le mucus cervical (vitamine $E$, cholestérol, phospholipides diacyles, sphingomyéline et plasmalogènes). Cette baisse, associée à un enrichissement lipidique concomitant des mucus cervicaux, est en faveur d'un efflux actif des lipides membranaires des spermatozoïdes vers les glycoprotéines des mucus cervicaux. Par ailleurs, lorsque les mucus cervicaux contenaient des polynucléaires neutrrophiles (PNN), les taux de phospholipides et la quantité de DAO produite par les spermatozoïdes étaient significativement plus élevés que lorsque les spermatozoïdes avaient migré dans des mucus cervicaux dépourvus de PNN. L'infiltration des mucus cervicaux par les PNN joue un rôle possiblement délétère sur les structures et fonctions des spermatozoïdes et inhiberait l'interaction physiologique entre les spermatozoïdes et le mucus cervical.

Mots clés : spermatozoïdes humains, lipides, dérivés actifs de l'oxygène, mucus cervical, polynucléaires neutrophiles

\section{Correspondance :}

Dr Nozha Chakroun Feki - Laboratoire d'Histologie-Embryologie et Biologie de la Reproduction, Faculté de Médecine de Sfax, Avenue Majida Bouleila, 3028 Sfax. Tunisie Tel 21674241888 - Fax 21674246217 Email nozhafeki@yahoo.fr 


\section{INTRODUCTION}

Le spermatozoïde humain, cellule hautement différenciée, est caractérisé par un contenu lipidique riche en acides gras polyinsaturés (AGPI). L'AGPI le plus abondant est l'acide docosahexaénoïque (DHA; $\mathrm{C} 22: 6, \mathrm{n}-3$ ) représentant environ $30 \%$ de tous les acides gras et $73 \%$ des AGPI [46, 54, $61,76]$. Dans le spermatozoïde humain, ces acides gras sont les constituants majoritaires des phospholipides membranaires. Les AGPI représentent des cibles privilégiées pour les dérivés actifs de l'oxygène (DAO).

Ainsi, le spermatozoïde humain est une cellule très vulnérable à la présence non contrôlée de dérivés actifs de l'oxygène compte tenu de sa richesse en AGPI [12, 47, 77]. Le déséquilibre de la balance prooxydants-antioxydants a des conséquences sur les capacités fonctionnelles des spermatozoïdes, essentiellement sur la mobilité, la réaction acrosomique, la fusion avec la membrane ovocytaire et l'intégrité du noyau du spermatozoïde $[1,7,49$, 70].

Cependant, au cours des dernières années il a été montré que des quantités faibles et contrôlées de DAO sont impliquées dans l'acquisition du pouvoir fécondant des spermatozoïdes humains $[8,27]$. Les seules études disponibles chez l'homme sur la composition lipidique des spermatozoïdes et leur susceptibilité vis-à-vis d'un stress oxydant ont été faites sur des spermatozoïdes épididymaires ou éjaculés $[9,28,45]$. Cependant, le destin physiologique des spermatozoïdes ne s'arrête pas à l'éjaculation. En effet, de la migration dans le mucus cervical à la fusion avec la membrane ovocytaire, le spermatozoïde doit maintenir un ensemble de capacités. Les micro-environnements successifs rencontrés dans le tractus génital femelle (TGF) jouent un rôle déterminant dans l'expression du pouvoir fécondant des spermatozoïdes $[20,48,51,77]$. S'il est établi qu'un faible niveau de production des DAO par les spermatozoïdes est impliqué dans l'expression de ses capacités fécondantes, il n'existe par contre que de rares données sur l'effet potentiel d'un déséquilibre de la balance prooxydants-antioxydants sur les fonctions du spermatozoïde humain au cours de sa migration dans le TGF.

Dans le présent article, nous ferons tout d'abord une revue bibliographique sur les modifications physiologiques du contenu lipidique des spermatozoïdes et leur susceptibilité au stress oxydant au cours de leur maturation dans les tractus génitaux masculin et féminin. Nous rapporterons brièvement ensuite une série de résultats originaux sur les modifications du contenu lipidique des spermatozoïdes au cours de la migration dans le mucus cervical et l'effet d'un stress oxydant induit par les polynucléaires neutrophiles du mucus cervical sur les caractéristiques spermatiques.

\section{COMPOSITION LIPIDIQUE DES SPER- MATOZOIDES HUMAINS EPIDIDYMAIRES ET EJACULES}

Après un cycle spermatogenétique, les spermatozoïdes prêts à quitter le testicule paraissent morphologiquement matures mais ils sont fonctionnellement immatures. En effet, ils n'ont acquis ni la mobilité ni la capacité de féconder un ovocyte. L'acquisition de la mobilité se fait au cours du transit dans l'épididyme [14]. Cependant, les spermatozoïdes fraîchement éjaculés n'ont pas acquis tout leur potentiel fécondant qui ne sera atteint qu'après une migration complète dans le TGF $[16,17,21,75]$.

Les modifications membranaires du spermatozoïde au cours de son transit dans le tractus génital male (TGM) et le TGF (pour ce dernier point, voir paragraphe III) dépendront essentiellement des échanges avec les différents milieux : fluide épididymaire, plasma séminal, mucus cervical, fluide tubaire et liquide folliculaire. Au niveau de l'épididyme, la membrane du spermatozoïde testiculaire subit des réarrangements moléculaires qualitatifs et spatiaux très importants : modification et perte de composants préexistants, addition de nouvelles glycoprotéines à partir des secrétions épididymaires et échanges de composants lipidiques [13, 40, 69]. Dans les spermatozoïdes, le contenu en cholestérol et en phospholipides diminue après le transit épididymaire [54] ; chez le rat, la baisse en phospholipides intéressant essentiellement les phosphatidyl choline et ethanolamine, est concomitante à un enrichissement en plasmalogènes surtout en plasmenylcholine, phospholipide majeur de la membrane des spermatozoïdes [18].

Concernant les acides gras, les spermatozoïdes perdent des acides gras saturés et s'enrichissent en acides gras à longues chaînes et polyinsaturés après migration dans l'épididyme [19]. L'acide palmitique, lié aux phospholipides est l'acide gras majeur dans les spermatozoïdes immatures, le DHA prédomine dans les spermatozoïdes matures [60]. Dans le sperme humain éjaculé, et après séparation des fractions cellulaires par gradient de densité de percoll, le taux de DHA était significativement plus élevé dans la fraction contenant des cellules germinales que celle contenant les spermatozoïdes [58].

La composition lipidique du spermatozoïde humain éjaculé est bien documentée $[12,39,56]$. Comparée aux cellules eucaryotes, la membrane du spermatozoïde humain possède trois particularités à signaler :

1) présence d'un taux élevé de plasmalogènes ;

2) taux élevé en acides gras polyinsaturés surtout l'acide docosahexaénoïque ; 

Alvarez et al. Hamamah et
Grizard et al.
1995 al. 1995
nmol/10 ${ }^{8} \mathrm{spz}$
nmol/10 ${ }^{7}$ spz
(1)
2000
$\mathrm{nmol} / 10^{8} \mathrm{spz}$
(4)

\section{Ollero et al. 2001 $\mathrm{nmol} / 10^{6} \mathrm{spz}$}
(3)
Notre étude
nmol/10 ${ }^{8}$
spermatozoïdes
(2)
(5)

$13,0 \pm 1,6 \quad 95 \pm 11$
$0,39 \pm 0,16$
$3,27 \pm 2,37$

220.9

DHA

$108,0 \pm 7,0$

Plasmalogènes

$20,0 \pm 0,2$
$282 \pm 4$

0,34

$0,6 \pm 0,1$

$22,3 \pm 2,5$

$95 \pm 11$

-

$-$

CL : Cholestérol ; PH : phospholipides ; AGS : acides gras saturés ; DHA : acide décosahexaénoïque

Les dosages étaient faits à partir :

- d'échantillons de spermes d'hommes volontaires dans les études (1), (3) et (4);

- d'échantillons de pools de spermes d'hommes volontaires dans l'étude (5);

- d'échantillons de spermes d'hommes infertiles normozoospermiques dans l'étude (2).

Les études (1) et (3) ont utilisé des spermatozoïdes sélectionnés (culot de gradient de densité).

Les études (2), (4) et (5) ont utilisé les spermatozoïdes totaux du sperme après séparation du plasma séminal.

3) un rapport cholestérol/phospholipides bas (Tableau 1). Ces particularités membranaires induisent une susceptibilité élevée à l'effet néfaste des DAO (voir plus loin).

\section{DERIVES ACTIFS DE L'OXYGENE ET SPERMATOZOIDE HUMAIN}

Le maintien d'un équilibre entre la quantité des DAO produits et l'efficacité des moyens antioxydants cellulaires et extracellulaires est nécessaire pour le fonctionnement normal d'une cellule. Si l'effet néfaste de l'oxygène et de ses dérivés actifs sur les fonctions cellulaires a été démontré depuis des dizaines d'années, ce n'est qu'au cours de ces dernières années que leur rôle physiologique dans la signalisation cellulaire a été évoqué.

\section{Rôle physiologique des DAO dans l'acquisition du pouvoir fécondant des spermatozoïdes humains}

Dans le spermatozoïde humain, des quantités faibles et contrôlées de DAO semblent contrôler l'acquisition du pouvoir fécondant [26]. La capacitation du spermatozoïde, étape préalable nécessaire à la fécondation, est définie par une série de transformations biochimiques et membranaires dans le tractus génital féminin [15]. Les mécanismes moléculaires de ce processus sont complexes et semblent impliquer un seuil minimum de DAO d'action spécifique produits par les spermatozoïdes eux mêmes. En effet, dans des conditions capacitantes, les spermatozoïdes produisent de faibles quantités d'anion superoxyde $\left(\mathrm{O}_{2}{ }^{\circ}\right)$ et d'hydro- peroxyde $\left(\mathrm{H}_{2} \mathrm{O}_{2}\right)$ [23]. L'ajout de $\mathrm{O}_{2}^{\circ}$ - ou de $\mathrm{H}_{2} \mathrm{O}_{2}$ accélère ce processus, la catalase et la superoxyde dismutase, enzymes qui catalysent la transformation respective de $\mathrm{O}_{2}{ }^{\circ}$ - et $\mathrm{H}_{2} \mathrm{O}_{2}$ en $\mathrm{H}_{2} \mathrm{O}$, le réduisent [24].

Le mécanisme de production $\mathrm{deO}_{2}{ }^{\circ}$-par le spermatozoïde humain est controversé. Il dépendrait d'une enzyme membranaire de type NADPH-oxydase [6, 63]. L'inhibition spécifique de cette enzyme diminue la phosphorylation des tyrosines au cours de la capacitation et le taux de capacitation des spermatozoïdes [53].

Le mécanisme d'action des DAO dans le processus de capacitation n'est pas précisément connu mais des résultats récents suggèrent leur rôle dans l'activation de l'adényl cyclase et la tyrosine kinase, au cours de la capacitation [8, $10]$.

La fixation spécifique du spermatozoïde capacité à la zone pellucide va permettre le déclenchement de la réaction acrosomique, processus d'exocytose du contenu de l'acrosome régulé par des quantités faibles et contrôlées de DAO [26]. Des concentrations faibles d' $\mathrm{H}_{2} \mathrm{O}_{2}$ favorisent la réaction acrosomique déclenchée in vitro par l'ionophore calcique A23187 et la pénétration des spermatozoïdes dans les ovocytes de Hamster [27]. La catalase inhibe ces phénomènes [27]. Il existe des discordances sur le rôle réel de $\mathrm{O}_{2}{ }^{\circ}$ - dans la réaction acrosomique $[27,37,38]$. En définitive et quel que soit le type de DAO impliqué, la majorité des données publiées indiquent l'existence d'une régulation redox de la capacitation et de la réaction acrosomique $[10,23]$. 
2. Susceptibilité des spermatozoïdes humains vis-àvis d'un stress oxydant et effet délétère des DAO

Le déséquilibre de la balance prooxydants/antioxydants crée un état de stress oxydant avec des conséquences notables sur les lipides membranaires et les autres constituants protéiques et nucléaires de la cellule $[2,4,25,36,49]$. Les dommages cellulaires sont généralement la conséquence d'attaque directe des constituants cellulaires par les DAO ou d'attaque indirecte par les produits réactifs de la peroxydation des AGPI de la membrane plasmique [71].

Le contenu important en AGPI de la membrane du spermatozoïde augmente sa vulnérabilité vis-à-vis de l'attaque par les DAO. Les DAO ont généralement une affinité importante à l'électron libre des doubles liaisons donnant naissance à des espèces moléculaires lipidiques instables. Ainsi se déclenche une chaîne de peroxydation lipidique qui se propage dans la membrane plasmique avec des conséquences sur sa fluidité et sur les fonctions spermatiques [46].

Les AGPI les plus sensibles aux DAO sont l'acide arachidonique et l'acide docosahexaénoïque (DHA) [35]. L'oxydation du DHA est un facteur majeur de baisse de la mobilité in vitro [12]. Le stress oxydant induit par les polynucléaires neutrophiles (PNN) a des effets néfastes sur les AGPI, se traduisant par une baisse du pourcentage de DHA dans les spermatozoïdes [77]. Les conséquences de cette baisse en DHA dans la membrane plasmique sont l'augmentation de la rigidité membranaire, la baisse de la mobilité des spermatozoïdes et la baisse de la capacité fusiogène avec la membrane ovocytaire [5]. Par ailleurs, les DAO ont la capacité d'induire une fragmentation de l'ADN et des oxydations des bases $[44,57,62]$.

L'origine des DAO dans le sperme humain est l'objet d'une controverse. Actuellement il est établi que les spermatozoïdes, les cellules germinales et les cellules phagocytaires contribuent toutes à la production des DAO avec des capacités et des mécanismes de production très différents (revus dans [9, 49]). Huszar et al. ont montré une relation entre la qualité du sperme et l'augmentation de la quantité de DAO produite par les spermatozoïdes due essentiellement à la présence d'excès cytoplasmiques reliés à un défaut de maturation $[43,57]$. Des travaux plus récents ont corroboré ces résultats $[28,31,33]$. Aitken et al. suggèrent que la production excessive de DAO par les spermatozoïdes avec excès cytoplasmique est induite par une enzyme cytoplasmique la glucose-6-phosphate déshydrogénase [1]. Les spermatozoïdes immatures ont la capacité de produire des niveaux élevés de DAO en réponse à une stimulation par un phorbol 12-myristate 13-acétate (PMA) [50]. Cependant, et comme en témoignent plusieurs études, les PNN activés représentent la source majeure de DAO dans le sperme $[3,64,72]$.
3. Rôle des polynucléaires neutrophiles dans le stress oxydant

Les PNN sont présents dans le tractus génital mâle et sont retrouvés dans quasiment tous les échantillons de sperme humain [68]. Cependant la signification clinique d'une infiltration importante de polynucléaires neutrophiles représente encore un sujet de controverse $[66,73]$. L'absence de signification physiopathologique de la leucospermie (concentration de PNN > 1 x106/ml; [74]) dans certaines études est probablement le reflet des propriétés antioxydantes du plasma séminal qui assure une protection vis-à-vis d'un stress oxydant induit par les DAO produits par les leucocytes $[11,67]$. Dans une étude récente, Saleh et al. [64] ont suggéré que les PNN stimulent ou induisent la production de quantités élevées de DAO par les spermatozoïdes eux mêmes : les spermatozoïdes des hommes infertiles ayant une leucospermie avaient un niveau de production spontanée et induite de DAO par le PMA significativement plus élevé que celui trouvé chez des hommes infertiles ou fertiles n'ayant pas de leucospermie. L'incubation des spermatozoïdes obtenus à partir de spermes non leucospermiques avec des leucocytes sanguins induit une production élevée de DAO par les spermatozoïdes à l'état natif ou après stimulation par le PMA [64].

\section{MODULATION DE LA COMPOSITION LIPIDIQUE DES SPERMATOZOIDES ET PHENOMENES OXYDATIFS AU COURS DE LEUR MIGRATION DANS LE TRACTUS GENITAL FEMININ}

L'interaction entre les spermatozoïdes et les fluides du tractus génital féminin (TGF) est une étape nécessaire pour l'expression du pouvoir fécondant des spermatozoïdes [48]. Dans ce domaine, la plupart des données ont été acquises chez l'animal car l'étude de ce processus in vivo chez l'homme présente des difficultés majeures techniques et éthiques. Chez l'homme, les conséquences de cette interaction sur la structure et la fonction des spermatozoïdes on été étudiées in vitro et de manière indirecte et séquentielle en utilisant chacun des fluides génitaux du TGF ou en recourant à des milieux synthétiques de composition moléculaire proche de celle des secrétions du TGF [20, 34, 41].

Les modifications du contenu lipidique et de la dynamique membranaire du spermatozoïde représentent le premier évènement inducteur des signaux intracellulaires de la capacitation [22, 52]. L'augmentation de la fluidité membranaire du spermatozoïde au cours de la capacitation des spermatozoïdes est la conséquence d'une diminution du rapport cholestérol/phospholipides suite à un efflux de cholestérol à partir de la membrane plasmique vers une 
protéine réceptrice $[30,42,55]$. L'albumine est un accepteur du cholestérol qui induit la capacitation in vitro [32].

Les modifications de la dynamique membranaire semblent agir directement ou indirectement sur les protéines membranaires telles celles constituant les canaux ioniques ou les enzymes intervenant dans le déclenchement des cascades de signalisation intracellulaire [59].

Il est donc très probable que la qualité des microenvironnements rencontrés par les spermatozoïdes dans le TGF soit un facteur important influençant la dynamique membranaire et l'expression des capacités fonctionnelles des spermatozoïdes.

Enfin, il n'existe à notre connaissance aucune donnée sur les dysfonctions spermatiques induites par des anomalies de la balance prooxydants/antioxydants dans le TGF lors d'une inflammation localisée, d'une endométriose ou d'autres processus pathologiques.

\section{RESUME DE NOTRE ETUDE}

Se basant sur les données de la littérature démontrant

1) le rôle néfaste d'un stress oxydant sur la composition lipidique des spermatozoïdes et sur les fonctions spermatiques, et

2) les modifications physiologiques de ces dernières dans le TGF, l'hypothèse de travail que le statut oxydatif du tractus génital féminin (TGF) constitue un modulateur de la structure et des capacités fonctionnelles des spermatozoïdes peut être raisonnablement faite.

Dans le but d'étudier cette question et d'explorer parallèlement les modifications du contenu lipidique des spermatozoïdes humains au cours de leur migration dans le TGF, nous avons initié une recherche sur la première étape de l'interaction des spermatozoïdes avec les microenvironnements du TGF, c'est-à-dire l'étape de migration des spermatozoïdes dans le mucus cervical. Deux objectifs ont été fixés : d'une part, étudier les modifications des caractéristiques fonctionnelles des spermatozoïdes humains éjaculés, de la composition en lipides membranaires et de la production des DAO après migration dans le mucus cervical et, d'autre part, comparer les caractéristiques des spermatozoïdes après migration dans le mucus cervical en fonction de la présence ou non de PNN dans ce milieu.

\section{Modifications du contenu lipidique des spermato- zoïdes après migration dans le mucus cervical}

\section{a) Matériel et Méthodes}

Nous avons utilisé et adapté la technique dite de «swimout» décrite par Zhu et al. [78] pour la sélection des spermatozoïdes après migration dans le mucus cervical. La Figure 1 illustre cette technique et résume les différentes étapes du protocole expérimental. Des échantillons de spermes de volontaires sains ont été recueillis et plusieurs pools ont été constitués. Par ailleurs, le mucus cervical de plusieurs femmes en période ovulatoire et volontaires pour participer à cette étude a été prélevé et sa qualité a été appréciée de manière standardisée en recourant au score de l'OMS [74]. Le contenu en PNN a été mesuré après liquéfaction et marquage à la peroxydase.

Nous avons utilisé une méthode originale pour le dosage des sous espèces moléculaires de phospholipides, du cholestérol et de la vitamine $\mathrm{E}$ par chromatographie liquide haute performance (CLHP) décrite par Thérond et al. [65]. Les taux de cholestérol, de vitamine $\mathrm{E}$, de plasmalogène, de sphingomyéline et des phospholipides diacyles contenant des acides gras polyinsaturés (1-palmitoyl-2-docosahexaenoyl-sn-glycero-3-phospholipide : 16:0/22:6; 1-palmitoyl-2-arachidonoyl-sn-glycero-3-phospholipide : 16:0/20:4 et le 1-stearoyl-2-docosahexaenoyl-sn-glycero3-phospholipide : 18:0/22:6) ont été déterminés sur chaque échantillon de sperme étudié, à l'état natif (l'ensemble de la population de spermatozoïdes) et sur la sous-population récupérée après migration dans le mucus cervical. Cette méthode a également été utilisée pour déterminer les profils lipidiques du mucus cervical, après liquéfaction de ce dernier, à l'état natif et après migration des spermatozoïdes (Figure 2).

\section{b) Résultats}

Comme attendu, la mobilité et les caractéristiques de vitesse des spermatozoïdes (Tableau 2) étaient significativement augmentées après migration $(\mathrm{p}<0,005)$. Nous avons démontré que la composition lipidique des spermatozoïdes baisse significativement après migration dans le mucus cervical (Figure 3). L'analyse lipidique des mucus cervicaux avant migration des spermatozoïdes indiquait que le cholestérol bien qu'en faible quantité, était le composant lipidique majeur (Figure 2). Surtout, nous avons démontré que les mucus cervicaux s'enrichissent en vitamine $\mathrm{E}$, en cholestérol et en phospholipides après migration des spermatozoïdes (Figure 2). Les modifications du contenu lipidique des spermatozoïdes après migration dans le mucus cervical semblent être en rapport avec un efflux actif de lipides sous l'action des glycoprotéines du mucus cervical. Cette hypothèse est soutenue par les modifications concomitantes du profil lipidique des mucus. Ce phénomène représenterait la première étape du processus de capacitation. Toutefois, l'hypothèse de la sélection d'une sous population des spermatozoïdes caractérisés par un contenu lipidique bas n'est pas exclue. Dans une étude récente de Force et al., la sous population de spermatozoïdes humains récupérée après sélection par test du swim-up était caractérisée par un contenu lipidique bas (cholestérol, sphingomyéline, phospholipides) comparé à l'ensemble de la 


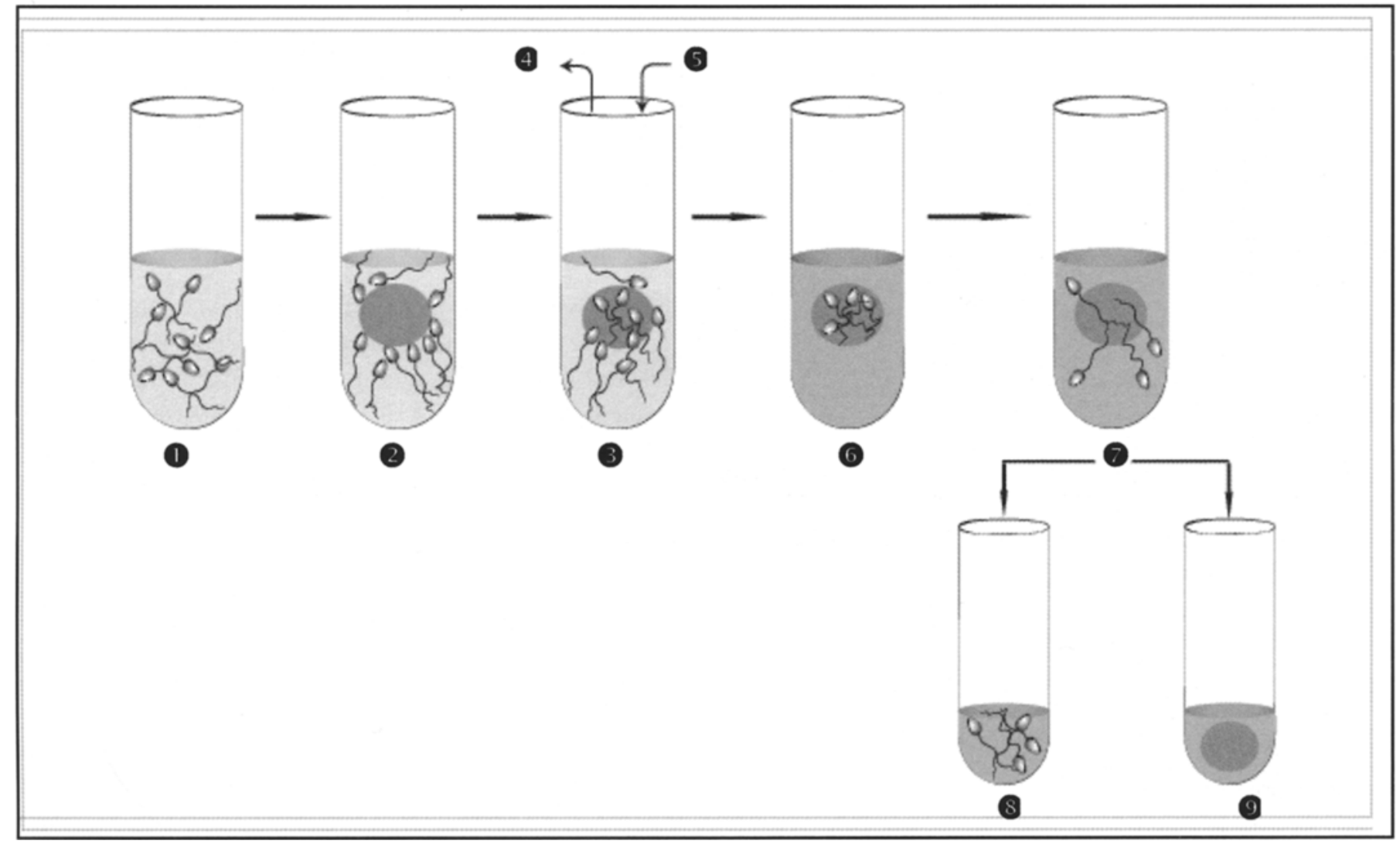

Figure 1 : Protocole expérimental.

(1) Préparation du pool de spermes ( 2 à 3 échantillons de spermes d'hommes volontaires).

(2) Déposition du mucus cervical au dessus du pool de spermes.

3 1ère incubation ( $37^{\circ} \mathrm{C}$; 25 min.) ; "swim-in" : les spermatozoïdes migrent dans le mucus cervical.

4 Elimination du sperme.

5 Injection puis aspiration avec une pipette pasteur du milieu de Earle ( 2 fois) autour du mucus cervical pour éliminer les spermatozoïdes qui n'ont pas pénétré dans le mucus cervical.

6 2ème incubation dans du milieu du Earle (37 ${ }^{\circ} \mathrm{C}$; 25 min.) ; "swim-out” les spermatozoïdes migrent du mucus cervical vers le milieu de Earle.

(6) Séparation du mucus cervical du milieu de Earle contenant les spermatozoïdes sélectionnés.

8 Liquéfaction du mucus cervical (broméline : $5 \mathrm{mg} / \mathrm{ml} ; 60 \mathrm{~min}$. ; $37^{\circ} \dot{\mathrm{C}}$ ) et récupération du surnageant après centrifugation (10 min. à $1200 \mathrm{~g}$ ). 

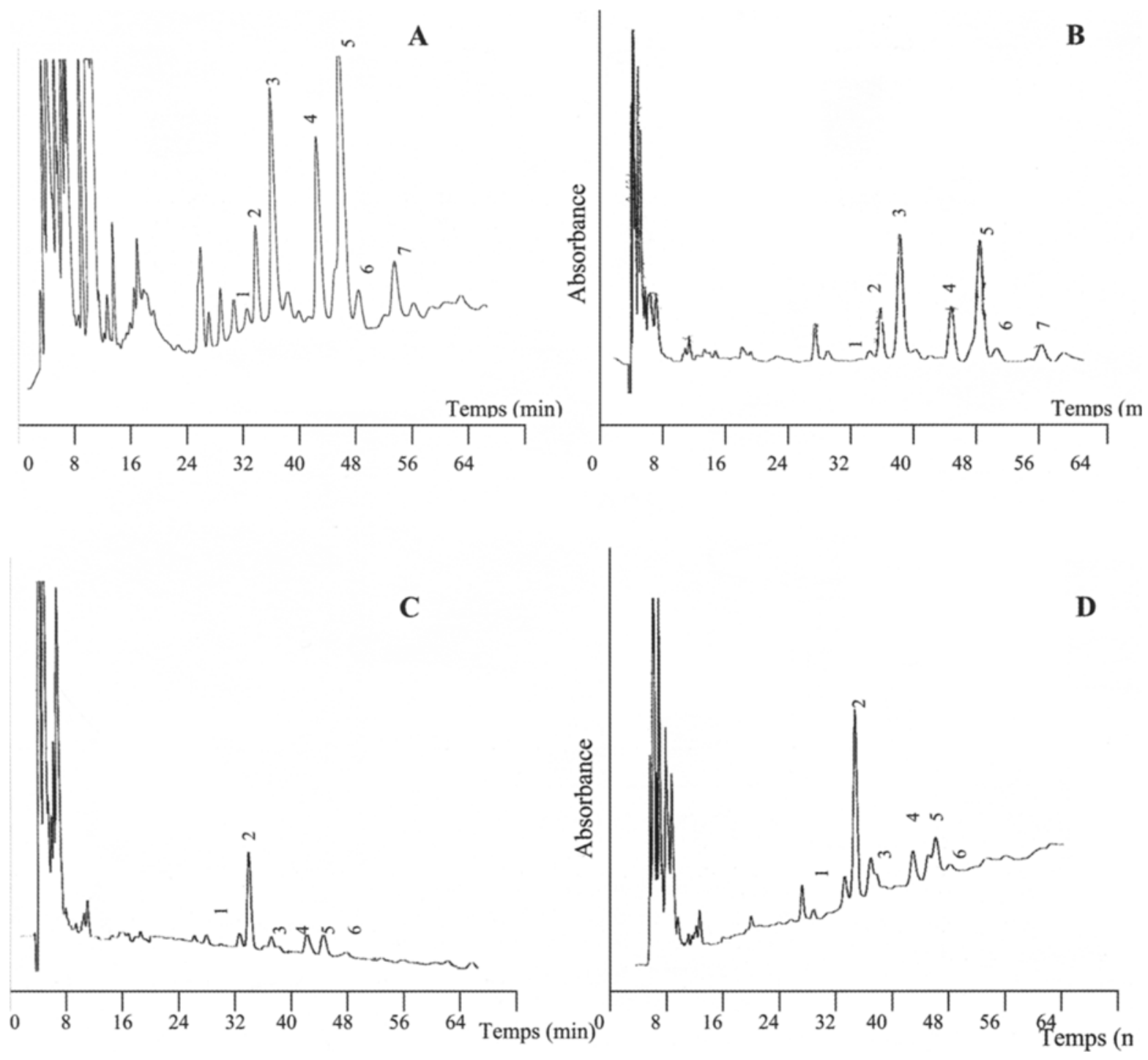

Figure 2 : Analyse par HPLC des lipides membranaires des spermatozoüdes humains et des mucus cervicaux.

A : Profil chromatographique des spermatozoïdes humains éjaculés.

B : Profil chromatographique des spermatozoïdes humains après migration dans les mucus ovulatoires.

C: Profil chromatographique des mucus cervicaux ovulatoires.

$D$ : Profil chromatographique des mucus cervicaux ovulatoires après migration des spermatozoüdes.

Dans $C$ et $D$, seuls les surnageants ont été dosés, les culots cellulaires récupérés après une forte centrifugation sont éliminés.

1: Vitamine $E$;

2: Cholestérol;

3: 1-palmitoyl-2-docosahexaenoyl-sn-glycero-3-phospholipide : 16:0/22:6;1-palmitoyl-2-arachidonoyl-sn-glycero-3-phospholipide :

16:0/20:4;

4: Plasmalogène ;

5: alkyl éther ;

6: Sphingomyéline;

7: 1-stearoyl-2-docosahexaenoyl-sn-glycero-3-phospholipide : 18:0/22:6. 

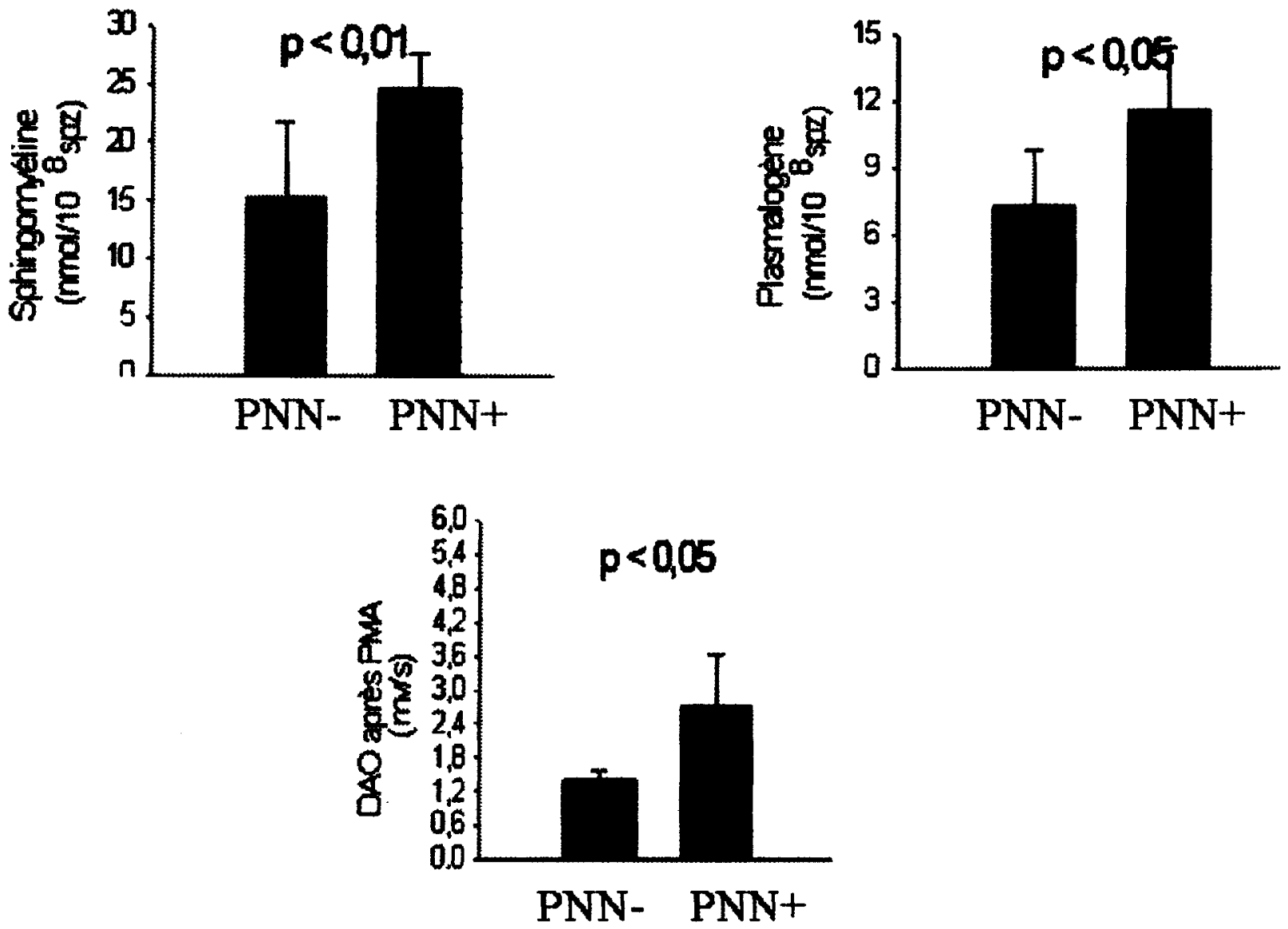

Figure 3 : Composition lipidique (sphingomyéline et plasmalogènes) et production des dérivés actifs de l'oxygène (DAO) après stimulation par le phorbol 12-myristate 13-acétate (PMA) dans les spermatozoüdes après migration dans le mucus cervical contenant ou non des polynucléaires neutrophiles (PNN). Tous les mucus étudiés étaient favorables à la migration des spermatozoüdes, (score OMS >12). PNN+ : PNN > $10000 / \mathrm{ml}$ de mucus $(n=4)$. PNN-: PNN < $10000 / \mathrm{ml}$ de mucus $(n=7)$.

Tableau 2 : Caractéristiques des spermatozoüdes avant et après migration dans le mucus cervical.

Pool de spermes $n=8$

$$
55,1 \pm 3,1(44,-76,0)
$$

$36,1 \pm 3,8(23,0-58,0)$

Mobilité Progressive (\%)

$74,0 \pm 2,1(65,0-84,0)$

$41,4 \pm 2,8(31,0-51,0)$
Spermatozoïdes après migration dans le mucus cervical $n=12$

$$
\begin{aligned}
& 76,6 \pm 4,0(47,0-92,0)^{a} \\
& 54,1 \pm 5,0(21,0-78,0)^{a} \\
& 82,7 \pm 3,0(75,0-97,0)^{a} \\
& 50,5 \pm 4,5(33,0-71,0)^{b}
\end{aligned}
$$

$a=p<0,005 ; b=p<0,01$ comparé aux spermatozoüdes du pool de spermes

Les mobilités ont été mesurées par un système automatisé (CASA).

La morphologie est appréciée selon la classification de David. 
population de spermatozoïdes à l'état natif [29]. Ces auteurs suggèrent que la population des spermatozoïdes sélectionnée par cette technique est formée de spermatozoïdes plus matures en se basant sur le fait qu'ils sont caractérisés en plus par une surface cytoplasmique réduite (activité créatine kinase faible).

\section{Caractéristiques spermatiques en fonction de la} présence ou non de polynucléaires neutrophyles (PNN) dans le mucus cervical (MC)

\section{a) Matériel et Méthodes}

Tous les mucus cervicaux utilisés dans notre étude permettaient la migration des spermatozoïdes et avaient des scores OMS supérieurs à 13. Ils étaient répartis arbitrairement en deux groupes : un premier groupe avec une concentration en PNN >10.000 par millilitre de $\mathrm{MC}$, et un deuxième groupe avec une concentration en PNN $<10.000$ par millilitre de MC (limite de détection des PNN dans le MC). La production radicalaire totale de suspensions de spermatozoïdes avant et après migration dans les mucus cervicaux, a été étudiée par chimiluminescence en utilisant le Luminol [74].

\section{b) Résultats}

La quantité des DAO produits par les spermatozoïdes après migration dans le mucus cervical était significativement diminuée par rapport à celle enregistrée dans les spermatozoïdes du pool. Par contre, la mobilité et la vitalité des spermatozoïdes n'étaient pas significativement différentes en fonction de la présence ou non de PNN dans le mucus. De plus, après migration dans des mucus cervicaux contenant des PNN la production des DAO par les spermatozoïdes après stimulation par le PMA était significativement augmentée (Figure 2). Ainsi, les PNN des mucus cervicaux semblent avoir un effet inducteur sur la production des DAO par les spermatozoïdes. Cette hypothèse est appuyée par les résultats très récents de Salah et al. [59]. En effet, dans cette étude, la leucospermie était associée à une capacité élevée de production de DAO par les spermatozoïdes, spontanément ou après stimulation par le PMA. L'incubation des spermatozoïdes obtenus à partir de spermes non leucospermiques avec des leucocytes sanguins induit une production élevée de DAO par les spermatozoïdes, de manière spontanée ou après induction par le PMA. Ces phénomènes, dont le mécanisme est totalement inconnu, pourraient impliquer les cytokines libérées par les PNN comme outils de signalisations cellulaires ayant comme réponse une augmentation de la production des DAO [59].

Enfin, nous avons démontré que les spermatozoïdes sélectionnés après migration dans un MC contenant des PNN avaient des taux de diacyles, de sphingomyéline et de plas- malogènes significativement plus élevés que ceux trouvés pour les spermatozoïdes sélectionnés par des mucus cervicaux sans PNN (Figure 2). Ces phénomènes probablement complexes pourraient avoir un impact direct sur l'expression du potentiel fécondant des spermatozoïdes ou sur la qualité de l'embryon, ou plus tard au cours du développement embryonnaire.

\section{CONCLUSION}

Le mucus cervical ovulatoire est une étape déterminante au cours de la migration du spermatozoïde humain dans le tractus génital féminin. Il joue un rôle certain dans la sélection d'une sous population de spermatozoïdes mobiles et un rôle très probable dans la dynamique des lipides membranaires de cette population de spermatozoïdes selon nos résultats préliminaires. Ce rôle semble être modulé par la qualité des mucus et particulièrement par la présence de polynucléaires neutrophiles. Ces phénomènes probablement très complexes et susceptibles d'avoir un impact sur l'expression du potentiel fécondant suggèrent l'intérêt de développements futurs de l'approche présente étudiant expérimentalement les spermatozoïdes sous l'angle de son interaction avec les milieux normaux ou pathologiques rencontrés dans le tractus génital féminin.

\section{REFERENCES}

1. AITKEN R.J. : The Amoroso Lecture. The human spermatozoon - a cell in crisis ? J. Reprod. Fertil., 1999, 115 : 1-7.

2. AITKEN R.J. : Free radicals, lipid peroxydation and sperm function. Reprod. Fertil. Dev., 1995, 7 : 659-669.

3. AITKEN R.J., BAKER H.W.G. : Seminal leucocytes : passengers, terrorists or good samaritans ? Hum. Reprod., 1995, 10 : 1736-1739.

4. AITKEN R.J., CLARKSON J.S. : Cellular basis of defective sperm function and its association with the genesis of reactive oxygen species. J. Reprod. Fertil., 1987, 81 : 459-469.

5. AITKEN R.J., CLARKSON J.S., FISHEL S. : Generation of reactive oxygen species, lipid peroxydation, and human sperm function. Biol. Reprod., 1989, 41 : 183-197.

6. AITKEN R.J., FISHER H.M., FULTON M. et al. : Reactive oxygen species generation by human spermatozoa is induced by exogenous NADPH and inhibited by the flavoprotein inhibitors diphenylene iodonium and quinacrine. Mol. Reprod. Dev., 1997, 47 : 468-482.

7. AITKEN R.J., GORDON E., HARKISS D. et al. : Relative impact of oxidative stress on the functional competence and genomic integrity of human spermatozoa. Biol. Reprod., 1998, 59 : 1037-1046.

8. AITKEN R.J., HARKISS D., KNOX W., PATERSON M., IRVINE D.S. : A novel signal transduction cascade in capacitating human spermatozoa characterised by a redox-regulated, c AMPmediated inductionof tyrosine phosphorylation. J. Cell Sci., 1998, $111:$ 645-656. 
9. AITKEN RJ., KRAUSZ C. : Oxidative stress, DNA damage and the Y chromosome. Reproduction, 2001, $122: 497-506$.

10. AITKEN R.J., PATERSON M., FISHER H., BUCKINGHAM D.W., DUIN M. : Redox regulation of tyrosine phosphorylation in human spermatozoa and its role in the control of human sperm function. J. Cell Sci., 1995, 108 : 2017-2025.

11. AITKEN R.J., WEST K., BUCKINGHAM D. : Leukocytic infiltration into the human ejaculate and its association with semen quality, oxidative stress, and sperm function. J. Androl., 1994, 15: 343-352.

12. ALVAREZ J.G., STOREY B.T. : Differential incorporation of fatty acids into and peroxidative loss of fatty acids from phospholipids of human spermatozoa. Mol. Reprod. Dev.,1995, $42: 334-346$.

13. AMANN R.P., HAMMERSTEDT R.H., VEERAMACHANENI D.N. : The epididymis and sperm maturation : a perspective. Reprod. Fertil. Dev., 1993, 5 : 361-381.

14. AMANN R.P., HAY S.R., HAMMERSTEDT R.H. : Yield, Characteristics, motility and cAMP content of sperm isolated from seven regions of ram epididymis. Biol. Reprod., 1982, 27 : 723733.

15. AUSTIN C.R. : Capacitation of spermatozoa. Int. J. Fertil., $1967,12: 25-31$.

16. AUSTIN C.R. : Observations on the penetration of the sperm into the mammalian egg. Aust. J. Res., 1951, 4 : 581-596.

17. AUSTIN C.R. : The capacitation of the mammalian sperm. Nature., $1952,170: 326$.

18. AVELDANO M.I., ROTSTEIN N.P., VERMOUTH N.T. : Lipid remodelling during epididymal maturation of rat spermatozoa. Enrichment in plasmenylcholines containing long-chain polyenoic fatty acids of the n-9 series. Biochem. J., 1992, $283: 235-$ 241.

19. AVELDANO M.I., ROTSTEIN N.P., VERMOUTH N.T. : Occurrence of long and very long polyenoic fatty acids of the n9 series in rat spermatozoa. Lipids. 1992, $27: 676-680$.

20. BARRATT C.L., COOKE I.D. : Sperm transport in the human female reproductive tract - a dynamic interaction. Int. J. Androl., $1991,14: 394-411$.

21. CHANG M.C. : Fertilization capacity of spermatozoa deposited into fallopian tubes. Nature, 1951, $168: 697-698$.

22. CROSS N.L. : Role of cholesterol in sperm capacitation. Biol. Reprod., 1998, $159:$ 7-11.

23. DELAMIRANDE E., GAGNON C. : Capacitation associated production of superoxide anion by human spermatozoa. Free Rad. Biol. Med., 1995, 18 : 487-495.

24. DELAMIRANDE E., GAGNON C. : Human sperm hyperactivation and capacitation as parts of an oxidative process. Free Radic. Biol. Med., 1993, 14 : 157-166.

25. DE LAMIRANDE E., GAGNON C. : Reactive oxygen species and human spermatozoa. I. Effects on the motility of intact spermatozoa and on sperm axonemes. J. Androl., 1992, 13 : 368-378.

26. DE LAMIRANDE E., JIANG H., ZINI A., GAGNON C. : Reactive oxygen species and sperm physiology. Rev. Reprod., 1997, 2 : 48-54.
27. DELAMIRANDE E., TSAI C., HARAKAT A., GAGNON C. : Involvement of reactive oxygen species in human sperm acrosome reaction induced by $\mathrm{A} 23187$, lysophosphatidylcholine, and biologic fluid ultrafiltrates. J. Androl., 1998, 19 : 585-594.

28. FISHER H.M., AITKEN R.J. : Comparative analysis of the ability of precursor germ cells and epididymal spermatozoa to generate reactive oxygen metabolites. J. Exp. Zool., 1997, 277 : 390400 .

29. FORCE A., GRISARD G., GIRAUD N., MOTTA C., SION B., BOUCHER D. : Membrane fluidity and lipid content of human spermatozoa selected by swim-up method. Int. J. Androl., 2001, $24: 327-334$.

30. GAMZU R., YOGEV L., PAZ G., YAVETZ H., LICHTENBERG D. : Reduction of sperm cholesterol : phospholipid ratio is a possible mechanism for enhancement of human sperm binding to the zona pellucida following incubation with phosphatidylcholine liposomes. Biol. Reprod., 1997, 57 : 539-546.

31. GIL-GUZMAN E.. ORELLO M.. LOPEZ M.C. et al. : Differential production of reactive oxygen species by subsets of human spermatozoa at different stages of maturation. Hum. Reprod., 2001, $16: 1922-1930$.

32. GO K.J., WOLF D.P. : Albumin-mediated changes in sperm sterol content during capacitation. Biol. Reprod., 1985, 32 : 145153.

33. GOMEZ E., BUCKINGHAM D., BRINDLE J., LANZAFAME F., IRVINE S.D., AITKEN R.J. : Development of image analysis system to monitor the retention of residual cytoplasm by human spermatozoa : correlation with biochemical markers of cytoplasmic space, oxidative stress and sperm function. J. Androl., 1996, $17: 276-287$.

34. GOULD J.E., OVERSTREET J.W., HANSON F.W. : Assessment of human sperm function after recovery from the female reproductive tract. Bio1. Reprod., 1984, 31 : 888-894.

35. GRIVEAU J.F., DUMONT E., RENARD P., CALLEGARI J.P., LE LANNOU D. : Reactive oxygen species, lipid peroxidation and enzymatic defence systems in human spermatozoa. $J$. Reprod. Fertil., 1995, 103 : 17-26.

36. GRIVEAU J.F., LELANNOU D. : Reactive oxygen species and human spermatozoa : physiology and pathology. Int. J. Androl., $1997,20: 61-69$.

37. GRIVEAU J.F., RENARD P., LE LANNOU D. : An in vitro promoting role for hydrogen peroxide in human sperm capacitation. Int. J. Androl., 1994, $17:$ 300-307.

38. GRIVEAU J.F., RENARD P., LE LANNOU D. : Superoxide anion production by human spermatozoa as a part of the ionophore-induced acrosome reaction process. Int. J. Androl., $1995,18: 67-74$.

39. GRIZARD G., BAUCHART D., BOUCHER D. : Separation and quantification of cholesterol and major phopholipid classes in human semen by high-performance liquid chromatography and light scattering detection. J. Chromatography B, 2000, 740 : 101107.

40. HAIDL G., OPPER C. : Changes in lipids and membrane anisotropy in human spermatozoa during epididymal maturation. Hum. Reprod., 1997, 12 : 2720-2723. 
41. HAMAMAH S., LANSON, M., BARTHELEMY, C. et al. : Analysis of the lipid content and the motility of human sperm after follicular fluid treatment. Andrologia, 1995, 27 : 91-97.

42. HOSHI K., AITA T., YANAGIDA K., YOSHIMATSU N., SATO A. : Variation in the cholesterol/phospholipid ratio in human spermatozoa and its relationship with capacitation. Hum. Reprod., 1990, 5 : 71-74.

43. HUSZAR G., SBARACIA M., VIGUE L., MILLER D.J., SHUR B.D. : Sperm plasma membrane remodeling during spermiogenetic maturation in men : relationship among plasma membrane beta 1, 4-galactosyltransferase, cytoplasmic creatine phosphokinase, and creatine phosphokinase isoform ratios. Biol. Reprod., 1997, $56: 1020-1024$.

44. IRVINE D.S., TWIGG J.P., GORDON E.L., FULTON N., MILNE P.A., AITKEN R.J. : DNA integrity in human spermatozoa : relationships with semen quality. J. Androl., 2000, $21: 33$ 44.

45. JONES R. : Plasma membrane structure and remodelling during sperm maturation in the epididymis. J. Reprod. Fertil., Suppl., $1998,53: 73-84$.

46. JONES R., MANN T., SHERINS R. : Peroxidative breakdown of phospholipids in human spermatozoa, spermicidal propreties of fatty acid peroxides and protective action of seminal plasma. Fertil. Steril., 1979, $31:$ 531-537.

47. JONES R., MANN T. : Lipid peroxides in spermatozoa ; formation, role, of plasmalogen and physiological significance. Proc. Roy. Soc. London B, 1976, 193 : 317-333.

48. KATZ D.F., DROBNIS E.Z., OVERSTREET J.W. : Factors regulating mammalian sperm migration through the female reproductive tract and oocyte vestments. Gamete Res., 1989, 22 : 443-469.

49. KIM J.G., PARTHASARATHY S. : Oxidation and the spermatozoa. Sem. Reprod. Endocrinol., 1998, 16 : 235-239.

50. KRAUSZ C., MILLIS C., ROGERS S., TAN S.L., AITKEN R.J. : Stimulation of oxidant generation by human sperm suspensions using phorbol esters and formyl peptides : Relation ships with motility and fertilization in vitro. Fertil. Steril., 1994, 62 : 599605 .

51. LAMBERT H., OVERSTREET J.W., MORALES P. et al. : Sperm capacitation in the human female reproductive tract. Fertil. Steril., 1985, $43: 325-327$.

52. LANGLAIS J., KAN F.W., GRANGER L., RAYMOND L., BLEAU G., ROBERTS K.D. : Identification of sterol acceptors that stimulate cholesterol efflux from human spermatozoa during in vitro capacitation. Gamete Res., 1988, 20 : 185-201.

53. LECLERC P., DELAMIRANDE E., GAGNON C. : Regulation of protein-tyrosine phosphorylation and human sperm capacitation by reactive oxygen derivatives. Free Radic. Biol. Med., $1997,22: 643-656$.

54. LENZI A., GANDINI L., MARESCA V. et al. : Fatty acid composition of spermatozoa and immature germ cells. Mol. Hum. Reprod., 2000, $6: 226-231$

55. LIN Y., KAN F.W. : Regionalization and redistribution of membrane phospholipids and cholesterol in mouse spermatozoa during in vitro capacitation. Biol. Reprod., 1996, 55 : 1133-1146.
56. LIN D.S., CONNOR W.E., WOLF D.P., NEURINGER M., HACHEY D.L. : Unique lipids of primate spermatozoa : desmosterol and docosahexaenoic acid. J. Lipid Res., 1993, 34 : 491-499.

57. OLLERO M., GIL-GUZMAN E., LOPEZ M.C. et al. : Characterization of subsets of human spermatozoa at different stages of maturation : implications in the diagnosis and treatment of male infertility. Hum. Reprod., 2001, $16: 1912-1921$.

58. OLLERO M., POWERS R.D., ALVAREZ J.G. : Variation of docosahexaenoic acid content in subsets of human spermatozoa at different stages of maturation : implications for sperm lipoperoxidative damage. Mol. Reprod. Dev., 2000, 55 : 326-334.

59. OSHEROFF J.E., VISCONTI P.E., VALENZUELA J.P., TRAVIS A.J., ALVAREZ J., KOPF G.S. : Regulation of human sperm capacitation by cholesterol efflux stimulated signal transduction pathway leading to protein kinase A mediated up regulation of protein tyrosine phosphorylation. Mol. Hum. Reprod., $1999,5: 1017-1026$.

60. PARKS J.E., HAMMERSTEDT R.H. : Development changes occurring in the lipids of ram epididymal spermatozoa plasma membrane. Biol. Reprod., 1985, 32 : 653-668.

61. POULOS A., DARIN-BENNETT A., WHITE I.G. : The phospholipids-bound fatty acids and aldehydes of mammalian spermatozoa. Comp. Biochem. Physiol., 1973, 46 : 541-549.

62. RAMOS L., WETZELS A. : Low rates of DNA fragmentation in selected motile human spermatozoa assessed by the TUNEL assay. Hum. Reprod., 2001, 16 : 1703-1707.

63. RICHER S.C., FORD W.C.A. : Critical investigation of NADPH oxidase activity in human spermatozoa. Mol. Hum. Reprod., $2001,3: 237-244$.

64. SALEH R.A., AGARWAL A., KANDIRALI E. et al. : Leukocytospermia is associated with increased reactive oxygen species production by human spermatozoa. Fertil. Steril., 2002, 78 : 1215-1224.

65. THEROND P., COUTURIER M., DEMELIER J.F., LEMONNIER F. : Simultaneous determination of the main molecular species of soybean phosphatidylcholine or phosphatidylethanolamine and their corresponding hydroperoxides obtained by lipoxygenase treatment. Lipids, 1993, $28: 245-249$.

66. THOMAS J., FISHEL S.B., HALL J.A., GREEN S., NEWTON T.A., THORNTON S.J. : Increased polymorphonuclear granulocytes in seminal plasma in relation to sperm morphology. Hum. Reprod., 1997, 12 : 2418-2421.

67. TOMLINSON M.J., BARRATT C.L., COOKE I.D. : Prospective study of leukocytes and leukocyte subpopulations in semen suggests they are not a cause of male infertility. Fertil. Steril., 1993, $60: 1069-1075$.

68. TOMLINSON M.J., WHITE A., BARRATT C.L., BOLTON A.E., COOKE I.D. : The removal of morphologically abnormal sperm forms by phagocytes : a positive role for seminal leukocytes ? Hum. Reprod., 1992, 7 : 517-522.

69. TOSHIMORI K. : Maturation of mammalian spermatozoa : modifications of the acrosome and plasma membrane leading to fertilization. Cell Tissue Res., 1998, 293 : 177-187.

70. TWIGG J., IRVINE D.S., HOUSTON P., FULTON N., 
MICHAEL L., AITKEN R.J. : Iatrogenic DNA damage induced in human spermatozoa during sperm preparation : protective significance of seminal plasma. Mol. Hum. Reprod., 1998, 4 : 439-445.

71. TWIGG J., FULTON N., GOMEZ E., IRVINE D.S., AITKEN R.J. : Analysis of the impact of intracellular reactive oxygen species generation on the structural and functional integrity of human spermatozoa : lipid peroxidation, DNA fragmentation and effectiveness of antioxidants. Hum. Reprod., 1998, 13 : 14291436.

72. WHITTINGTON K., FORD W.C. : Relative contribution of leukocytes and of spermatozoa to reactive oxygen species production in human sperm suspensions. Int. J. Androl., 1999, 22 : 229235.

73. WOLFF $\mathrm{H}$. : The biologic significance of white blood cells in semen. Fertil. Steril., 1995, 63 : 1143-1157.

74. WORLD HEALTH ORGANISATION (WHO) : Laboratory manual for the examination of human semen and semen-cervical mucus interaction. $2^{\text {nd }}$ ed. Cambridge, United Kingdom, The press syndicate of the University of Cambridge, 1999.

75. YANAGIMACHI R. : Fertility of mammalian spermatozoa: its development and relativity. Zygote, 1994, 2 : 371-372.

76. ZALATA A.A., CHRISTOPHE A.B., DEPUYDT C.E., SCHOONJANS F., COMHAIRE F.H. : The fatty acid composition of phospholipids of spermatozoa from infertile patients. Mol. Hum. Reprod., 1998, 4 : 111-118.

77. ZALATA A.A., CHRISTOPHE A.B., DEPUYDT C.E., SCHOONJANS F., COMHAIRE F.H. : White blood cells cause oxidative damage to fatty acid composition of phospholipids of human spermatozoa. Int. J. Androl., 1998, 21 : 154-162.

78. ZHU, J., BARRATT C.L., LIPPES J. et al. : The sequential effects of human cervical mucus, oviductal fluid, and follicular fluid on sperm function. Fertil. Steril., 1994, 61 : 1129-1135.

\section{ABSTRACT}

Human sperm lipid content and susceptibility to reactive oxygen species (ROS) before and after migration through human

cervical mucus

\author{
Nozha CHAKROUN FEKI, Patrice THEROND, \\ Pierre JOUANNET, Jacques AUGER
}

Spermatozoa are particularly susceptible to damage induced by ROS, especially as their plasma membrane contains large amounts of polyunsaturated fatty acids. Mammalian sperm cells develop the capacity to fertilise ova during transport in the male and female reproductive tracts. The nature and quality of the micro-environment of the female reproductive tract are important factors for sperm selection, capacitation and subsequent acrosome reaction. In vitro experiments using capacitating media have shown remodeling of the lipid composition of the sperm membrane during these steps and the same approaches have also shown that a low level of ROS was necessary. The oxidative status of the female genital tract is therefore certainly of primary importance for the physiological maturation of the sperm cell. It has been previously reported that an inappropriate oxidative balance in the male genital tract (ie, an excessive ROS production overwhelming all antioxidant strategies) impairs the structure and several functions of sperm cells. This phenomenon may arise in the female genital tract, but has never been investigated.

The present paper is a review of the literature on these subjects and also reports our results concerning the changes in semen lipid content during cervical mucus migration and the effect of cervical mucus polymorphonuclear (PMN) cells on sperm characteristics.

We showed that the sperm levels of vitamin E, cholesterol, phospholipids, sphingomyelin and plasmalogen assessed by HPLC decreased after migration through cervical mucus. These modifications were observed in parallel with lipid enrichment of the cervical mucus, suggesting an efflux of cholesterol and lipids from sperm cells. The spermatozoa recovered postmigration in the cervical mucus were characterised by low levels of the various lipid classes.

Spermatozoa that migrated in cervical mucus samples with a considerable quantity of polymorphonuclear leukocytes (PMN) also showed significantly increased levels of sphingomyelin, diacyl phospholipids and plasmalogens in comparison to spermatozoa that migrated in cervical mucus devoid of PMN. Finally, we also found that PMA-induced ROS production was significantly increased for spermatozoa treated with cervical mucus containing PMN.

Key words: human spermatozoa, lipids, reactive oxygen species, cervical mucus, polymorphonuclear leukocytes

Prix DEA, SALF 2002.

Manuscrit reçu : mai 2003 ; accepté juillet 2003. 